University of Windsor

Scholarship at UWindsor

2003

\title{
Hox Genes as Synchronized Temporal Regulators: Implications for Morphological Innovation
}

Michael J. Crawford

University of Windsor

Follow this and additional works at: https://scholar.uwindsor.ca/biologypub

Part of the Biology Commons

\section{Recommended Citation}

Crawford, Michael J., "Hox Genes as Synchronized Temporal Regulators: Implications for Morphological Innovation" (2003). Journal of Experimental Zoology Part B: Molecular and Developmental Evolution, 295B, 1, 1-11.

https://scholar.uwindsor.ca/biologypub/10

This Article is brought to you for free and open access by the Department of Biological Sciences at Scholarship at UWindsor. It has been accepted for inclusion in Biological Sciences Publications by an authorized administrator of Scholarship at UWindsor. For more information, please contact scholarship@uwindsor.ca. 
This is a preprint of an article published in: JOURNAL OF EXPERIMENTAL ZOOLOGY (MOL DEV EVOL) 295B:1-11 (2003)

\title{
Hox genes as synchronized temporal regulators: implications for morphological innovation
}

\author{
Michael Crawford ${ }^{1 *}$ \\ Dept. Biological Sciences University of Windsor, 401 Sunset, Windsor, \\ Ontario, N9B 3P4, CANADA
}

\begin{abstract}
In vertebrates, clusters of Hox genes express in a nested and hierarchical fashion to endow the embryo's segments with discrete identities. Later in development, members of the same gene family are employed again to pattern the limb, intestinal, and reproductive systems. A careful analysis of the morphologies of Hox mutant mice suggests that the genes provide qualitatively different cues during the specification of segments than they do during the development of more recently derived structures. In addition to the regulatory differences noted by others, the activity of Hox genes during specification of the vertebrate metameres in some recent deletion experiments is inconsistent with a role for them as strictly spatial determinants. On the contrary, the phenotypes observed are suggestive of a role for them as elements of a generic time-keeping mechanism. By contrast, the specification of more recent evolutionary structures appears to be more spatial andgene-specific. These differences in role and effect may suggest some simple mechanisms by which the Hox clusters operate, and rules by which gene networks can be diverted to create new structures over the course of evolution. Specific predictions and experiments are proposed. J. Exp.
\end{abstract}

Zool. (Mol. Dev. Evol.) 294:000-000, 2002. @ 2002 Wiley-Liss, Inc.

\section{Introduction}

It seems to be a recurring theme that when a modular network of genetic activity works well to orchestrate some process, it is often employed, either in part or as a whole, over and over again throughout evolution and development. In humans and mice, there are thirty-nine Hox genes that play a role in the development of the axial skeleton, limbs, genitalia, and the intestinal and reproductive tracts. The genes express in an overlapping hierarchy of expression domains, and in different tissues at different times, however our understanding of how they help to implement discrete developmental effects remains obscure. This conceptual limitation is exacerbated by the nature of the target genes which have been recently identified: although still rather few in number, some exhibit qualities which suggest that the downstream complexity of Hox gene activity may be indirectly conferred by the historical/spatial peculiarities of a cell's context at different times during development (Brodu et al., '02). Some examples of target genes include basic-FGF, rho, p53, and $p 21$ (Brodu et al., '02; Bromleigh and Freedman, '00; Care et al., '96; Raman et al., '00). Until recently, it appeared that Hox genes elaborated a spatial map, a code, according to which body segments differentiated. Theory and experiment meshed nicely when loss- and gain-of-function manipulations seemed to confirm that the genes could anteriorize or posteriorize developing body segments in a predictable fashion.

\footnotetext{
${ }^{1}$ Grant Sponsor: Natural Sciences and Engineering Research Council of Canada; Grant number 203549
} 


\section{CRAWFORD}

While most investigators would agree that different combinations of Hox genes are required to direct the differentiation of discrete morphological regions, two different views have emerged regarding the specificity of action of this family of transcription factors. One camp argues that Hox proteins, although compositionally distinct from each other, nevertheless act in a generic manner, and it is the number, expression domain, and timing of their expression, not the particular Hox protein translated that may be important in modulating morphological differentiation (Crawford, 1995, Zakany et al, 1996). The other camp contends upon the basis of experimental evidence that individual Hox genes encode products that are sufficiently distinct as to confer a functionally unique role to each during development (for example, Zhao and Potter, 2001). These two views are not as irreconcilable as they might appear at first inspection: the interpretive differences lie primarily in the stage of development that is the focus of investigation. Hox genes play fundamentally different roles throughout development. These differences are a reflection of two features of developmental regulation: the degree to which the sub-system undergoing patterning is evolutionarily derived, and the degree to which the entire Hox apparatus has been recruited to perform a particular function.

In the arthropods and vertebrates, Hox gene activity is inextricably bound to metamerism. If one accepts that the Hox complex initially evolved to specify attributes of the antero-posterior axis in these organisms, several interesting possibilities arise which have implications both for Hox gene specificity, and the means by which modules of genetic activity can be redeployed over the course of evolution. It is a confounding accident of history that our understanding of Hox gene function began to unfold first in Drosophila, and that many of the concepts developed subsequently coloured analysis of vertebrate Hox gene activity. However, there are substantial functional and operational differences in the way Hox genes act in vertebrates and in Drosophila. Firstly, the cluster has duplicated in vertebrates, and this might confer additional roles that impinge upon morphology. Secondly, qualitative differences of Hox gene function and activity are likely amplified by both mechanical and temporal attributes that differentiate vertebrate from Drosophila development. For example, in Drosophila the Hox genes act soon after cellularization, and within a

\footnotetext{
* Correspondence to: Michael J. Crawford, Dept. Biological Sciences, University of Windsor, 401 Sunset, Windsor, Ont. N9B 3P4 Canada. Email:mcrawfo@uwindsor.ca

Received 8 July, 2002, Accepted 25 September, 2002.

Published online in Wiley InterScience (www.interscience.wiley.com)
}

context where many other hierarchies of genetic activity have already subdivided the syncitial stage blastoderm into discrete presumptive segments. By contrast, in vertebrate embryos (and indeed some other arthropods), $H o x$ genes act sequentially upon a progressively emerging rostro-caudal organization and segmentation of body plan. Perhaps these distinctions underlie the differences that inactivation of Hox genes create in fruitflies and vertebrates. In vertebrates the inactivation or ectopic expression of Hox genes can lead to transformation of axis specification only incrementally in one direction or another: a developing cervical vertebra can be transformed into an anterior thoracic vertebra, but not into a sacral one. Moreover, when vertebral segments are transformed, they nevertheless develop in an axially contiguous context - thoracic vertebra 1 will always form beside thoracic vertebra 2 and never beside 7 (see Crawford, '95 for review). By contrast, manipulation of fruitfly Hox genes can lead to major reorganization along the antero-posterior axis. For example, deletion of the caudally expressed bithorax complex of genes completely abrogates development of the abdominal segments and a segment approximating thoracic segment 2 is re-iterated instead. If ultrabithorax is added back into these deletion mutants, abdominal segments 2-8 are transformed into reiterated abdominal segment 1, and abdominal segment 9 remains intact (Lawrence and Morata, '94; Wolpert et al., '98). One reason for the more limited repertoire of transformations achievable in Hox mutant vertebrates may lie with the duplicated nature of the clusters: overlapping responsibilities and redundant function might render the vertebrate axis resistant to profound remodeling when only one or two of the genes are inactivated. For this reason, there has been considerable effort paid to the compound deletion of paralogous genes (ie; Hoxa4, b4, $c 4$ and $d 4$ ), and to entire clusters. Thirdly, another difference between fruitfly and vertebrate mutant phenotypes is that the muddled specification of segments by Hox genes can lead to legs growing out of heads in fruitfly (Kaufman et al., '90), but similar such radical transformations don't occur in vertebrates.

The current view that vertebrate Hox genes play a generic role as spatial determinants during anteroposterior axis differentiation is inconsistent with experimental evidence. Instead, the mutant phenotypes are more easily explained if one takes a more global view: it is possible that it is not the individual genes but the synchronized "unwinding" of the four Hox gene clusters that is important. If one posits that individual genes are more like elements in a larger developmental clock or metronome, and that perturbations of one gene 
likely disturb the activity of the remainder of the cluster, several problematic mutant phenotypes are explicable.

Under normal circumstances, since the vertebrate body plan emerges rostro-caudaly, the when and where of Hox gene expression are linked - cells receive a cue, and act in a contextually appropriate manner. As we shall see, the implications of a temporal versus spatial role are subtle but profound, and a generic role for the genes as elements of a metronome might also go some way to explaining why homeotic transformations in vertebrates tend to be in units of only one or a few segments anteriorly or posteriorly, and not more profound as seems to be possible in Drosophila. Having said that, it is also clear that parts of the Hox complex have become uncoupled from their normal regulatory context to perform additional, and more specific roles later during elaboration of systems like the limbs and reproductive tract. In doing so, they have been removed from their role as time-keepers or counting mechanisms. The pattern of this functional uncoupling and re-deployment of genes reveals why some modes of genetic change and morphological innovation are more easily created and fixed during evolution (Larsen, '97, in press).

\section{Homeobox Gene Specificity}

There can be no dispute that different homeobox genes encode proteins that are structurally distinct. However, in certain contexts, it has been apparent for several years that some homeobox genes are functionally interchangeable as long as the timing and domains of their expression are similar. For example, gooseberry and paired, are normally transcribed at different times during fruit fly development, however ectopic expression of one can have the effect of rescuing the null mutant phenotype of the other ( $\mathrm{Li}$ and Noll, '94). In addition, the knockout phenotypes of the two murine engrailed loci En-1 and $E n 2$, are morphologically and functionally distinct. Indeed, the proteins only share $55 \%$ amino acid identity, and they are responsible for different aspects of brain and limb patterning. Nevertheless, it appears that during brain development an En-1 null mutant phenotype can be rescued if an additional En-2 coding region is ectopically expressed under the control of an En-1 promoter (Hanks et al., '95). In other words, as long as the timing and domain of expression is preserved, the genes appear to be functionally interchangeable during this phase of development. If homeobox genes, by virtue of their structural and functional differences are supposed to confer distinct attributes to different body segments during development, how is it that they can occasionally function interchangeably? The recent Hox gene literature has focused considerable attention upon this problem, and the solution seems to depend upon how investigators have elected to establish their criteria for evaluation of generic $v s$. specific modes of action.

An analysis of murine Hox mutant phenotypes had earlier suggested that these transcription factors might provide generic cues and be functionally interchangeable (Crawford, '95). Elegant experimental evidence substantiated this view when it was discovered that Hox genes can rescue the mutant vertebral phenotypes of their paralogues when inserted ectopically into an appropriate regulatory context (Zakany et al., '96, Greer et al, '00). Furthermore, even non-paralogous genes retain functional equivalence during axis specification (Zhao and Potter, '01). More recently, the homeobox of an "anterior" Hox gene, Hoxa4, was inserted to replace the divergent homeobox of a posterior gene, Hoxal1. Although the chimeric gene elicited anomalous development later in development, with regard to elaboration of the antero-posterior axis, the swap was inert (Zhao and Potter, '02) These latter two experiments serve to illustrate the minimal semantic difference between the generic $v s$. specific action points of view: resistance to the notion that Hox genes can act generically arises from the observation that while axial attributes might be relatively normal in ectopically "rescued" mutants, other morphological features are not. For example, in Hoxa-11 and a-13 substituted mice, the antero-posterior axis is specified normally, but the limbs and reproductive tracts in females are not (Zhao and Potter, '01). Similar disparities between homeoboxmediated specification of antero-posterior axis and lateral structures is evident in the engrailed knock-in mice mentioned earlier - rescue of the brain mutant phenotype did not extend to rescue of anomalous limb development (Hanks et al., '95). Recently, evidence has been presented to suggest that paraxial and lateral mesoderm employ Hox cues in a different manner and that the two positional specification processes and their respective Hox "codes" may be different (Nowiki and Burke, '00). This observation is substantiated by evidence that the Hox clusters are regulated axially by ancient clustercentered elements, and laterally by more recently acquired regulatory regions, some of which might lie 5 , or 3' to the cluster (van der Hoeven et al., '96, Hérault et al, '99, Kmita et al., '00, Spitz et al., '01). It seems reasonable then, to separate the effects of Hox gene misexpression upon axial specification from those effects seen in more recently derived structures.

Hox genes and axial periodicity 


\section{CRAWFORD}

If Hox genes are interchangeable or playing a generic role in antero-posterior axis specification, what is the nature of the cues that they confer to the emerging neural tube and somites? There are two experimental thrusts where we might look for hints: one is direct and the other is speculative but may offer an explanation of unexpected phenotypes seen in mice where entire $H o x$ clusters have been deleted. Both series of experiments suggest an intimate link between Hox gene activity patterns and segmentation, and both place an emphasis upon the provision of temporal rather than spatial cues. Direct evidence linking patterns of Hox gene expression to temporal regimentation comes from Hox expression patterns prior to segmentation and perturbations in segmentation-impaired mice (Zakany et al., '01). Immediately prior to somite formation, there is a burst of Hox gene activity: the genes are transcribed in a dynamic and transient manner. Segmentation involves many gene products among which numbers RBPJk - an effector of the Notch signaling pathway, and a molecule that is likely to play an important role in the periodic production of somites from the pre-somitic mesoderm. In RBPJk mutant mice, not only is somitogenesis perturbed, but transcriptional bursts of Hox gene activity are altered. This suggests a direct link between specification of the antero-posterior axis by Hox genes and the activity of the hairy/notch segmentation clock (Zakany et al., '01). In addition, FGF8 modulates the "segmentation clock": it alters the ability of cells to regulate positional attributes when transplanted, it prevents presomitic mesoderm from segmenting, and it changes the boundaries of Hox gene expression (Dubrulle et al., '01). At present, nobody really knows what these "bursts" of Hox transcriptional activity signify. On the basis of the target genes identified to date, we might presume that Hox proteins modulate the activity of genes with influence upon growth such as $F G F$, or upon the cell cycle and differentiation such as Rho, p53, and p21 (Brodu et al., '02; Bromleigh and Freedman, '00; Care et al., '96; Raman et al., '00).

A more speculative link comes from unexpected phenotypes seen in mice where alternative technologies have been employed to knockout individual Hox genes, and where entire clusters of Hox genes have been ablated. The older knockout technology employed a neomycin selection marker that was inserted into a gene to render it inactive. The consequences of this insertion in the context of Hox clusters appears to have been a little more complex than first envisaged: gene disruption by insertion of the neomycin resistance cassette can have unanticipated and artifactual consequences, and the results are not always the same if a gene is knocked out using alternative recombinase-based approaches (Fiering et al., '93; Rijli et al., '94, Beckers and Duboule, '98). The reason for these discrepancies resides in the nature of Hox gene regulation - the genes share regulatory elements, and insertion of the neomycin resistance cassette interposes an insulator between normally contiguous spans of chromatin. One part of a Hox cluster can be effectively insulated from activity in the other by the neomycin cassette, and the adjacent "intact" genes express anomalously. The benefit of Cre recombinase-based approaches is that the selection marker, the neomycin resistance gene, is ultimately removed and only a very small recombinase binding motif is left behind. This has had important ramifications for Hox inactivation studies. For example, when a regulatory region situated between Hoxd11 and Hoxd12 was deleted using neomycin cassette insertion methods, patterns of Hox gene expression were altered, and mutant phenotypes arose. When the intergenic region was deleted by means of Cre recombinase, neither Hox gene expression patterns nor morphologies were aberrant (Beckers and Duboule, '98). This last point is important to the proposal that I will advance shortly. Firstly it demands that we regard the activity of Hox genes, minimally, within the context of expression patterns rendered by an entire cluster. Secondly it begs the question: if the genes are functionally interchangeable, and substantial functional inter- and intra-cluster redundancy exists, why should minor expression deviations caused by insertion of a neomycin cassette prove problematic for somite and neural specification?

If anyone had asked a vertebrate developmental biologist what the consequences for development would be were an entire vertebrate Hox cluster to be removed, chances are that they would likely have answered by outlining profound morphological deficits. Suemori et al., ('00) and Medina-Martinez et al., ('00) both express surprise at the phenotypes, but for unexpected reasons. To date, three separate large-scale deletions have been performed, and the resultant morphologies have been unexpectedly mild. In the case of the Hoxd and Hoxb cluster deletions, antero-posterior patterning anomalies arose, but they were no more severe than the sum of anomalies likely to be seen if genes were knocked out individually (Medina-Martinez et al., '00, Spitz et al., '01). Deletion of most of the Hoxb complex had no effect upon heterozygotes, and even homozygous nulls were deemed remarkable for exhibiting transformations that were limited in extent to anterior transformations of only one segment (Medina-Martinez et al., '00). In the case of the Hoxc cluster deletion, there appeared to be little effect upon axial specification - vertebral elements were neither absent, nor transformed to anterior or 
posterior fates although thoracic vertebrae 10 and 12 may have been slightly altered - axial development was otherwise almost completely normal (Suemori et al., '00). At the very least, one might have expected to see the sum of transformations elicited by the deletion of genes deleted individually. Such was not the case. These conflicting results beg two questions, both of which relate to the role of Hox genes in patterning of periodic structures. First, why did the two of the cluster deletions only perturb development in an incrementally antero-grade manner, and second, why did a third deletion experiment fail to elicit any axial anomalies when individual genes knocked out from the same cluster are known to have profound effects (for examples see, Le Mouellic et al., '92, Suemori et al., '95; Saegusa, '96)?

In addition to the Hoxc cluster deletion phenotype (or lack thereof), there is another compound mutation that has been studied which is inconsistent with a role for the Hox genes in delimiting strict spatial cues. When investigators attempted to eliminate the confounding effects of redundancies of action, they interbred mice mutant for $H o x c 8, b 8$ and $d 8$ to produce compound mutants deleted for the paralogous genes. Curiously, against the background of Hoc-8/Hoxd-8 null mutants, the effect of Hoxb-8 deletion was to partially rescue the mutant phenotype (van Den Akker et al., '01). Why was the triple mutant phenotype less, not more severe?

The answer may be surprisingly simple: Hox clusters might act to lend generic temporal cues to the developing axial skeleton: in effect, each cluster may be operating as a sort of simple metronome to lend regular and periodic cues to the pre-somitic mesoderm. How the genes do this might be as simple as altering cell cycle kinetics via regulation of p53, p21 etc. periodically rendering mesodermal and neural tissues sensitive to context-specific differentiation cues. Consider the ramifications a temporal role would have for explaining the varied mutant phenotypes. Interposition of a genetic insulator into a cluster in the form of a neomycin selection cassette would have the effect not only of inactivating a targeted gene, but also of prohibiting the normal manner in which the cluster unfolds its products. Adjacent genes, removed from normal regulatory influences, might be induced to activate in a temporally and spatially inappropriate manner: effects upon adjacent genes are known to occur (Rijli et al, '94). If the rate of progressive Hox cluster activation is perturbed, and the cluster is acting as a sort of metronome, then it will fall out of synchrony with the other clusters unless there is substantial regulatory crosstalk between them (Fig 1a). Ablation of paralogous genes might have less effect if all paralogues are removed than if only one or two are removed presuming that the remaining genes (adjacent and later-activating) act generically and are not differentially affected between clusters. Similarly, complete inactivation of a cluster could be innocuous if the "metronomes" enjoy functional redundancy - it may be less damaging to remove a redundant metronome than to have it ticking out of synchrony with its partners (Fig 1 b). By the same token, a partial rescue of phenotype by the superimposition of a Hoxb-8 mutant upon the Hoxc- $8 / d-8$ nulls might reflect the consequences of impairing three clusters so that they fall out of normal activity in the same manner - no cluster is forced out of synchrony relative to the others (the a-8 paralogue is absent from the Hoxa cluster). Although the clusters cannot operate in normal fashion, they at least are similarly hobbled - the metronomes might miss a beat, but carry on in relative synchrony thereafter (Fig 1c).

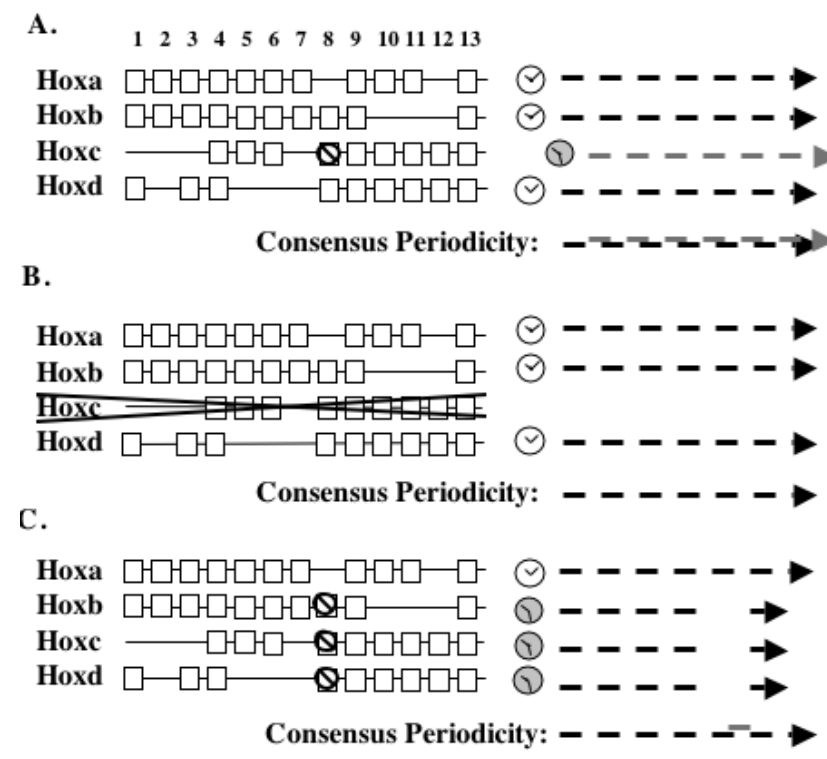

Fig. 1. Hox gene clusters as generic metronomes.

A) Disruption of a single Hox gene by introduction of a neomycin resistance marker insulates adjacent sequences from normal interaction. The pace at which the cluster "unfolds" is thrown out of synchrony with respect to the other clusters. This causes patterning problems which manifest as a homeotic transformation. B) Ablation of an entire cluster has the effect of removing a redundant metronome. Few axis deficits will arise as long as the deleted cluster is not required for the other clusters to operate normally. C) Ablation of paralogous genes might have less effect if all paralogues are removed than if only one or two are removed presuming that the remaining genes (adjacent and later-activating) act generically and are not differentially affected between clusters.

This leaves a conundrum though: the Hoxc cluster deletion was possible without gross effect upon 


\section{CRAWFORD}

the development of the antero-posterior axis, but the deletion of the $b$ and $d$ clusters was not. Why? The answer may lie in two directions. Firstly, the Hoxd and $b$ deletions were not complete - the $d l$ and the $b 13$ genes remained intact (Medina-Martinez et al., '00, Spitz et al., '01). Secondly, and most important, there are considerable structural differences that exist between the four mammalian Hox gene clusters.

The Hoxc gene cluster is quite different from the others in that it lacks the first three genes namely, the paralogues Hoxcl, c2, and $c 3$. Relatively little is known about how Hox genes are regulated, however, $H o x b 1, a 3, d 3, c 4$, and $d 4$ appear to auto-regulate as their protein products bind to their own promoter elements (McGinnis et al., '90, Arcioni et al., '92, Popperl et al., '92, Popperl et al., '95, Saleh et al, '00, Manzanares et al., '01). There are also indications of cross-talk between genes in the cases of Hoxa3, d3, $c 4$, and $b 4$ (Arcioni et al, '92, Manzaneres et al., '01). Moreover, the way that the Hoxbl enhancer is modulated is contingent upon the presence of the proteins $\mathrm{Pbx}, \mathrm{Hoxb} 1$, and an assemblage of other factors that include histone deacetylase (Saleh et al., '00). This early association with histone deacetylase indicates that there is a reasonable chance that activation of the early 3' Hox genes invokes changes in chromatin structure: acetylation/deacetylation of histones locally would have obvious repercussions for the rate and manner in which the rest of a Hox complex was "unpackaged" and activated. Lacking the first 3 Hox gene paralogues, the Hoxc complex is unlikely to be subjected to, or to effect changes in, the expression of genes from the other clusters in quite the same way. For example, deletion of the Hoxc cluster is unlikely to have a direct effect upon the way that the other clusters unfold during the early phase of Hox gene activation - it lacks the paralogues to interact with Hoxal, 2, 3, Hox b1, 2 3, and Hoxd1 and 3. By contrast, the deletion of any of the other clusters could result in aberrant activation kinetics for the remainder. In summary, some clusters are less likely to interact with the others during the early phases of activation: deletion of the Hoxc cluster is unlikely to have repercussions upon the early phases of activity of the others.

Similarly, deletion of all three of the Hox8 paralogues would have the effect of entirely removing a cue for posteriorization. This is why anteriorized transformations still occur in the triple mutant mice. This creates a potential conceptual problem though, because if the genes are acting in a generic manner, three of the clusters will come up one cue short to contribute to the last segments that need to be specified (Fig 1c). There are two possible answers to this problem. First, the missing cues could be regenerated by intercalation (Crawford, '95; Beck et al., '99). Second, the genes could be so generic in nature that the only intact cluster, the Hoxa cluster, is able to provide the final cue necessary to complete a countdown.

The clusters, save Hoxc, seem to be interdependent for early synchronous and progressive activation. The only circumstance where axial specification is forced off its trajectory is likely to be when one of the four "clocks" is thrown out of synchrony with the others. Mutual interactions between clusters would rapidly regulate anomalous behaviour, but an artifact of the perturbation could remain in the form of an anterior or posterior transformation. As long as removal of a gene or cluster does not interfere with the synchronized activity of the others, then there is no reason to expect that a mutant phenotype will arise during antero-posterior axis specification. By contrast, removal of large parts, but not all, of the Hoxb and $d$ clusters would likely have ramifications upon the manner in which the remaining genes in each cluster behave, and in how normally interacting paralogues might express. Finally, this perspective does not preclude morphological changes in more recently derived structures that redeploy Hox genes to direct local development.

\section{Hox clusters: clocks with redundant gears}

The Hoxc cluster-deleted mice died shortly following birth. A respiratory deficit is suspected (Suemori et al., '00). Nevertheless, we are still left with a big problem: given that deletion of the Hoxc cluster has no effect upon antero-posterior patterning, are we to conclude that none of the Hoxc genes are critical to axial specification? What do the genes do? How can they be dispensable given that the four clusters have been so highly conserved over a vast span of evolutionary time? The presence of the cluster in all tetrapods examined to date still suggests great antiquity. This paradoxical state of affairs is, I would argue, easier to understand if the clusters play a role in canalizing the temporal mechanics of axis development, and if the function of the Hoxc complex has been superimposed upon an older, already robust network.

Firstly, despite its age, the vertebrate Hoxc cluster is likely the most recently to have duplicated from the ancestral complex (Bailey et al., '97). Based upon the sequences of the Hox and neighboring genes, Bailey et al., ('97) deduced that the four complexes arose from an ancestral complex in three serial duplications, beginning with the Hoxd or $a$ clusters, and finishing with the Hoxb and $c$ clusters (Fig. 2). Both the $a$ and $d$ clusters are very ancient, and identifying whether $a$ or $d$ is more 
related to the ancestral complex is problematic (Ruddle et al., '99; Kim et al., '00). The functions of the Hoxc cluster have likely not integrated to the same extent as the older complexes, and the Hox clusters clearly play overlapping roles. Moreover, a duplicated cluster might be expected to operate in a milieu less constrained by bureaucratic linkages. It would offer itself and the parental cluster room to diverge as long as the ancestrally required functions were preserved between the two of them. Clusters could drop genes with impunity as long as either the parental or daughter cluster still retained functionality, and indeed, it is a general feature of Hox cluster evolution that as clusters duplicate, genes are lost (Chiu et al, '02) (Fig. 2). For example, if clusters $b$ and $c$ really did derive from a common ancestral cluster (Bailey et al., '97), it should not be surprising that between them they still constitute all thirteen paralogous genes (Fig. 2). For that matter clusters $a$ and $d$, which also diverged during one of the series of duplication events, together cover them all too, but two of three remaining cluster combinations $-a$ with $b$ and $c$ with $d$-do not, serving perhaps, as a nice confirmation of the postulated order of duplication. As a secondary consequence, removal to a remote site endowed with novel enhancers might bring regulatory novelties to the cluster and confer the possibility of new functions - such as a role in the elaboration of lung or diaphragm development.

\section{Specific Predictions}

If the Hox clusters really do act more as metronomes than as spatial selectors, then there are specific and testable hypotheses that we can consider. First, if the postulated order of cluster duplication is correct and individual Hox genes have been lost only where functional redundancy remains intact in a parent cluster, then deletion of "orphaned" paralogues might be expected to have a bigger effect than if duplicated ones are removed. Obviously, Cre recombinase deletion would have to be employed to test this hypothesis since the neomycin cassette technology gives problematic results (Fiering et al, '93). It is worth noting that the context of the Cre/lox deletion of Hoxd-13 regulatory regions (which manifests no morphological effect on axis differentiation) exists in a situation where there are three other Hox 13 paralogues to compensate (Beckers et al., '98). Better still, knock-in replacement strategies which substitute an inactivated gene with a pointmutation for the endogenous gene would remove some of the concern which would be engendered by manipulations of cluster conformational integrity. Since it is entirely possible that adjacent genes are regulated by elements that reside within their neighbor's coding sequence, even Cre recombinase strategies could elicit artifactual phenotypes. Moreover, the production of numerous antisense transcripts from at least one Hox gene suggests that deletion approaches might engender unforeseen consequences (Hsieh-Li et al., '95).

Second, if the clusters evolved to confer resistance to the confounding influences of a variable environment, then heat shock-induced segment anomalies will occur more readily during those phases of development when there are fewer duplicated paralogues available to constrain deviations from the normal pattern of antero-posterior axis specification. Furthermore, the degree of deviation from the norm should be inversely proportional to the number of paralogues available to rectify a potential patterning problem.

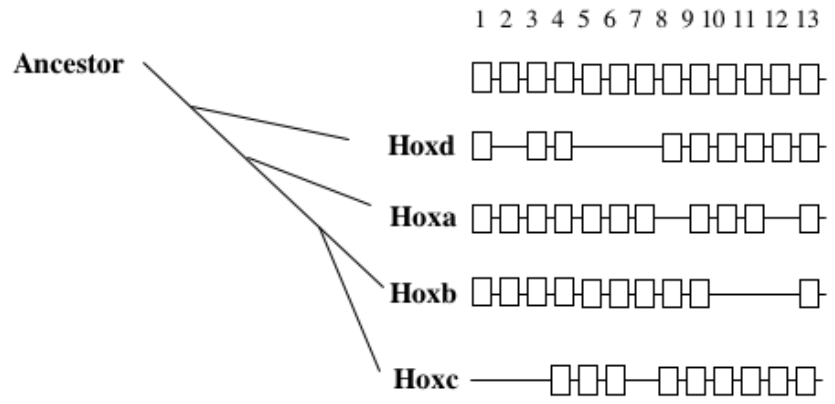

Fig.2. Hox cluster ancestry, redundancy of action, and conservation of paralogous complement.

Hox gene clusters duplicated in serial fashion, and subsequently lost individual paralogues. The diagram is not to scale, and is intended to illustrate that the Hoxd and $a$ clusters and the Hoxb and $c$ clusters group together the closest. Moreover, the clusters which are the most closely related, also possess between them, the full complement of Hox genes. (For a complete and accurate phylogenetic analysis see Bailey et al., '97). Whether the $a$ or $d$ cluster is the most closely related to the common ancestor has more recently become a matter of some ambiguity (Ruddle et al, et al., '99).

Third, the "rule of posterior prevalence" -where posterior Hox genes reset the developmental agenda established by anterior genes - needs re-testing in vetebrates. A critical control experiment has not yet been performed, namely the ectopic expression of an anteriorexpressing gene in a posterior domain. Although anterior genes and their promoter regions have been transplanted, with the exception of a homeobox swap experiment to create a chimeric product, anterior genes have not been transplanted to a regulatory context specifically appropriate to a posterior gene (Kmita et al., '00; Kondo and Duboule, '99; Zhao and Potter, '02). If the Hox genes 


\section{CRAWFORD}

are playing a spatial patterning role, the posterior expression of an anterior gene should have a phenotypic effect since it will be unable to dominate according to the rule of posterior prevalence. Alternatively, if the genes play a truly generic role, and the timing of expression is all that counts, then the anterior gene should function as a perfect replacement in a posterior regulatory context. Moreover, if the gene is instead inserted into the complex as a supernumerary posteriorly regulated gene, the additional cue should advance the segment specification clock and posteriorize somites despite its normal activity of providing an anterior cue.

Fourth, when duplicated genes or clusters are co-opted to a new developmental role, the genes most likely to acquire this functionality are those which operate under the least selective pressure, namely those genes which are in the most recently duplicated cluster, and those genes which sustain a role in tandem with a redundantly functional paralogue. In other words, introduction of new enhancer elements will cause the least confusion when newly acquired behaviour can be compensated for by a redundant paralogue. A case in point is provided by work in which the Hoxa 3 and $d 3$ genes have been altered. Each gene has a distinct mutant phenotype, however, Hoxd 3 plays a role in axial development, while Hoxa3 normally does not (Greer et al., '00). The restriction of Hoxa3 to stimulate development of a thymus and hyoid bone does not mean that it has lost its ability to regulate axial development if expressed in the appropriate context: If a Hoxa 3 coding region is expressed under the control of a Hoxd3 promoter in Hoxd 3 mutant mice, the mutant phenotype is rescued (Greer et al., 2000).

Lastly, deletion of the entire Hoxc cluster should have less of a phenotypic effect than deletion of all but one. Since an orphaned Hoxc gene would lack the context appropriate to normal "unfolding" of the Hoxc complex, its activity would be chronologically inappropriate. This would likely cause synchronization problems, and the amplitude of effect would be dependent partially upon the extent to which the "orphaned" Hoxc gene interacted with the remaining genes on other clusters.

\section{Why Retain Redundant Clocks And How Do Hox Gene Bureaucracies Modulate Morphological Change?}

Larsen's wonderful metaphor for gene regulation and activity as the product of an interaction between bureaucrats and workers illustrates how morphological innovation might follow the dissociation of modules of genetic interaction from their normal context. In describing the way that patterning is regulated, Larsen does a rough accounting and suggests that the number of genetic bureaucrats (signal receptors, transducers, and gene activator/repressors) is disproportionately large relative to the number of "worker" genes that implement cellular activity and differentiation (Larsen, '97, in press). Moreover, she points to the tendency for conserved gene networks to function in diverse roles and indicates that in evolutionary terms, it is likely easier to shuffle and redeploy larger assemblages of "bureaucratic" activity (signal transduction pathways), than to innovate new ones incrementally from scratch. Larsen argues that innovation would most easily occur through the shuffling of responsive elements either at the beginning or at the end points of a signal cascade (Larsen, '97, in press). This metaphor has utility in our analysis here, but to the list that includes beginning and end-point shuffling, we should add dissociation of genetic modules from "normal" constraints.

Generally speaking, when a bureaucracy enlarges, it also becomes more cumbersome and prone to inertia. Operational characteristics will come to limit the repertoire for interaction between bureaucrats and the actual effectors of the specific function they were designed to implement and regulate. In other words, the means of ensuring fidelity to an objective simultaneously becomes the means of limiting the degree to which constituent elements can enjoy the latitude to innovate. Eventually, regulatory and responsive networks become so intermeshed that the possibility for profound innovation is dampened. This makes sense when one of the objectives of the enterprise is to lend a process or developmental trajectory a robust quality which will ensure fidelity of (re-)creation. This is as true for large and heavily regulated corporations and governments as it is for genes and their products.

The program of antero-posterior axis specification in vertebrates seldom alters because the system has evolved to be robust. It is built to ensure morphological invariance despite changes in scale and physical environment (for an interesting and prescient discussion revisit Cooke, '78, and also see Cooke and Zeeman, '76, Elsdale et al., '76, and Primmett et al., '89, for the effects of temperature fluctuation upon vertebral specification). Redundant signals and interdependent regulation of activity (bureaucratic regulation) ensure that the Hox genes are linked to the progress of segmentation, and that the clusters behave to refine their own activity and that of each other. In sum, bureaucrats (for example receptors or transcription factors, in this case Hox genes) and workers (metabolic or cytoskeletal proteins), may, over the course of natural selection, have developed a robust but inertia-prone system for reliably 
producing morphological characteristics despite temperature or metabolic fluctuations. Now it appears that only relatively minor morphological changes can be elicited along the antero-posterior axis when Hox gene activity is perturbed. This point is emphasized when one considers that zebrafish exhibit no more morphological complexity than other vertebrates despite having two additional Hox gene clusters (Prince et al., 1998).

There are, however, ways to circumvent bureaucratic/regulatory inertia. Major leaps in morphological innovation are more likely to devolve from portions of larger and older gene regulatory networks which are removed from the context of their former constraints. Gene duplication has provided for two, superficially paradoxical morphological endpoints. Duplicated clusters may have evolved to ensure morphological invariance within the context of anteroposterior patterning, arguably one of the single most important events of embryogenesis. Gene duplication, however, also introduces a degree of latitude for subsets of these genes to acquire new attributes because at the level of individual genes, evolutionary constraints are relaxed. The introduction of remote enhancers over the course of evolution has permitted Hox genes to escape the temporal straightjacket normally imposed during antero-posterior axis specification, and to emerge to play new roles in different contexts, such as the elaboration of limb buds, or reproductive tracts. In this context, a thoughtful study of gene duplication events, and in particular of the duplication of entire clusters, will offer us a unique entré to study the effects of relaxed constraints upon the evolution of both genes and the morphologies that they help to direct.

\section{Acknowledgments}

Thanks are due to Drs. E. Larsen, A. Hubberstey and A. Ali, as well as F. KhosrowShahian and two anonymous reviewers for critical reading and suggestions.

\section{Literature Cited}

Arcioni L, Simeone A, Guazzi S, Zappavigna V, Boncinelli E, Mavilio F. 1992. The upstream region of the human homeobox gene $H O X 3 D$ is a target for regulation by retinoic acid and $H O X$ homeoproteins. EMBO J. 11: 265-277.

Bailey WJ, Kim J, Wagner GP, Ruddle FH. 1997. Phylogenetic reconstruction of vertebrate Hox cluster duplications. Mol Biol Evol 14: 843-53.

Beck F, Chawengsaksophak K, Waring P, Playford RJ Furness JB (1999) Reprogramming of intestinal differentiation and intercalary regeneration in $\mathrm{Cdx} 2$ mutant mice. Proc Natl Acad
Sci U S A Vol. 96, pp. 7318-23.

Beckers J, Duboule D. 1998. Genetic analysis of a conserved sequence in the HoxD complex: regulatory redundancy or limitations of the transgenic approach? Dev Dyn 213: 1-11.

Brodu V, Elstob PR Gould AP. 2002. abdominal A specifies one cell type in Drosophila by regulating one principal target gene. Development 129: 2957-63.

Bromleigh VC Freedman LP. 2000. p21 is a transcriptional target of HOXA10 in differentiating myelomonocytic cells. Genes Dev 14: 2581-6.

Care A, Silvani A, Meccia E, Mattia G, Stoppacciaro A, Parmiani G, Peschle C Colombo MP. 1996. HOXB7 constitutively activates basic fibroblast growth factor in melanomas. Mol Cell Biol 16: 4842-51.

Chiu CH, Amemiya C, Dewar K, Kim CB, Ruddle FH Wagner GP. 2002. Molecular evolution of the HoxA cluster in the three major gnathostome lineages. Proc Natl Acad Sci U S A 99: 5492-7.

Cooke J. 1978. Somite abnormalities caused by short heat shocks to pre-neurula stages of Xenopus laevis. J. Embryol. Exp. Morphol. 45: 283-294.

Cooke J, Zeeman EC. 1976. A clock and wavefront model for control of the number of repeated structures during animal morphogenesis. J. Theor. Biol. 58: 455-476.

Crawford M. 1995. Transformations in null mutants of Hox genes: do they represent intercalary regenerates? Bioessays 17: 106573 .

Dubrulle J, McGrew MJ, Pourquie O. 2001. FGF signaling controls somite boundary position and regulates segmentation clock control of spatiotemporal Hox gene activation. Cell 106: 219-32.

Elsdale T, Pearson M. 1979. Somitogenesis in amphibia. J. Embrol. Exp. Morphol. 53: 245-267.

Fiering S, Kim CG, Epner EM, Groudine M. 1993. An "in-out" strategy using gene targeting and FLP recombinase for the functional dissection of complex DNA regulatory elements: analysis of the beta-globin locus control region. Proc Natl Acad Sci U S A 90: 8469-73.

Greer JM, Puetz J, Thomas KR, Capecchi MR. 2000. Maintenance of functional equivalence during paralogous Hox gene evolution. Nature 403: 661-5.

Hanks M, Wurst W, Anson-Cartwright L, Auerbach AB, Joyner AL. 1995. Rescue of the En-1 mutant phenotype by replacement of En-1 with En-2. Science 269: 679-82.

Herault Y, Beckers J, Gerard M, Duboule D. 1999. Hox gene expression in limbs: colinearity by opposite regulatory controls. Dev Biol 208: 157-65.

Hsieh-Li HM, Witte DP, Weinstein M, Branford W, Li H, Small K Potter SS. 1995. Hoxa 11 structure, extensive antisense 


\section{CRAWFORD}

transcription, and function in male and female fertility. Development 121: 1373-85.

Kaufman TC, Seeger MA Olsen G. 1990. Molecular and genetic organization of the antennapedia gene complex of Drosophila melanogaster. Adv Genet 27: 309-62.

Kim CB, Amemiya C, Bailey W, Kawasaki K, Mezey J, Miller W, Minoshima S, Shimizu N, Wagner G Ruddle F. 2000. Hox cluster genomics in the horn shark, Heterodontus francisci. Proc Natl Acad Sci U S A 97: 1655-60.

Kmita M, Kondo T, Duboule D. 2000. Targeted inversion of a polar silencer within the HoxD complex re- allocates domains of enhancer sharing. Nat Genet 26: 451-4.

Kondo T Duboule D. 1999. Breaking colinearity in the mouse HoxD complex. Cell 97: 407-17.

Larsen E (in press) Genes, form and the tempo of evolution. In Muller G. and Kaufmann S. (eds.). Origination of organismal form: beyond the gene in developmental and evolutionary biology. MIT Press, Cambridge, MA, Vienna Series in Theoretical Biology.

Larsen E. 1997. Evolution of development: the shuffling of ancient modules by ubiquitous bureaucracies. In: Lumsden C, Brandt W, Trainor L, editors. Physical Theory in Biology. Singapore: World Science. P 431-444.

Lawrence P Morata G. 1994. Homeobox genes: their function in Drosophila segmentation and pattern formation. Cell 78: 181189.

Le Mouellic H, Lallemand Y, Brûlet P. 1992. Homeosis in the mouse induced by a null mutation in the Hox-3.1 gene. Cell 69: 251-264.

Li X, Noll M. 1994. Evolution of distinct developmental functions of three Drosophila genes by acquisition of different cisregulatory regions. Nature 367: 83-7.

Manzanares M, Bel-Vialar S, Ariza-McNaughton L, Ferretti E, Marshall H, Maconochie MM, Blasi F, Krumlauf R. 2001. Independent regulation of initiation and maintenance phases of Hoxa3 expression in the vertebrate hindbrain involve auto- and cross- regulatory mechanisms. Development 128: 3595-607.

McGinnis N, Kuziora MA, McGinnis W. 1990. Human Hox-4.2 and Drosophila Deformed encode similar regulatory specificities in Drosophila embryos and larvae. Cell 63: 969-976.

Medina-Martinez O, Bradley A, Ramirez-Solis R. 2000. A large targeted deletion of Hoxb1-Hoxb9 produces a series of singlesegment anterior homeotic transformations. Dev Biol 222: 7183.

Nowicki JL, Burke AC. 2000. Hox genes and morphological identity: axial versus lateral patterning in the vertebrate mesoderm. Development 127: 4265-75.
Pöpperl H, Bienz M., Studer M., Chan, S-K, Aparicio S., Brenner S., Mann R.S., Krumlauf R. 1995. Segmental expression of Hoxb-1 is controlled by a highly conserved autoregulatory loop dependent upon exd/pbx. Cell 81: 1031-1042.

Pöpperl H, Featherstone MS. 1992. An autoregulatory element of the murine Hox-4.2 gene. EMBO J. 11: 3673-3680.

Primmett DR, Norris WE, Carlson GJ, Keynes RJ, Stern CD. 1989. Periodic segmental anomalies induced by heat shock in the chick embryo are associated with the cell cycle. Development 105: 119-30.

Prince VE, Joly L, Ekker M, Ho RK. 1998. Zebrafish hox genes: genomic organization and modified colinear expression patterns in the trunk. Development 125: 407-20.

Raman V, Martensen SA, Reisman D, Evron E, Odenwald WF, Jaffee E, Marks J Sukumar S. 2000. Compromised HOXA5 function can limit p53 expression in human breast tumours. Nature 405: 974-8.

Rijli FM, Dolle P, Fraulob V, LeMeur M, Chambon P. 1994. Insertion of a targeting construct in a Hoxd-10 allele can influence the control of Hoxd-9 expression. Dev Dyn 201: 36677.

Ruddle FH, Amemiya CT, Carr JL, Kim CB, Ledje C, Shashikant CS Wagner GP. 1999. Evolution of chordate hox gene clusters. Ann N Y Acad Sci 870: 238-48.

Saegusa H, Takahashi N, Noguchi S, Suemori H. 1996. Targeted disruption in the mouse Hoxc-4 locus results in axial skeleton homeosis and malformation of the xiphoid process. Dev. Biol. 174: 55-64.

Saleh M, Rambaldi I, Yang XJ, Featherstone MS. 2000. Cell signaling switches HOX-PBX complexes from repressors to activators of transcription mediated by histone deacetylases and histone acetyltransferases. Mol Cell Biol 20: 8623-33.

Spitz F, Gonzalez F, Peichel C, Vogt TF, Duboule D, Zakany J. 2001. Large scale transgenic and cluster deletion analysis of the HoxD complex separate an ancestral regulatory module from evolutionary innovations. Genes Dev 15: 2209-14.

Suemori H, Takahashi N, Noguchi S. 1995. Hoxc-9 mutant mice show anterior transformation of the vertebrae and malformation of the sternum and ribs. Mech. Dev. 51: 265-273.

Suemori H, Noguchi S. 2000. Hox C cluster genes are dispensable for overall body plan of mouse embryonic development. Dev Biol 220: 333-42.

van Den Akker E, Fromental-Ramain C, de Graaff W, Le Mouellic H, Brulet P, Chambon P Deschamps J. 2001. Axial skeletal patterning in mice lacking all paralogous group 8 Hox genes. Development 128: 1911-21.

van der Hoeven F, Zakany J, Duboule D. 1996. Gene transpositions in the HoxD complex reveal a hierarchy of regulatory controls. Cell 85: 1025-35. 


\section{Hox GENES, SYNCHRONIZATION, AND MORPHOLOGY}

Wolpert L, Beddington R, Brockes JP, Jessel T, Lawrence PA Meyerowitz E (1998) Principles of Development. Current Biology/Oxford University Press, New York, pp. 484.

Zakany J, Gerard M, Favier B, Potter SS, Duboule D. 1996. Functional equivalence and rescue among group 11 Hox gene products in vertebral patterning. Dev Biol 176: 325-8.

Zakany J, Kmita M, Alarcon P, de la Pompa JL, Duboule D. 2001.
Localized and transient transcription of Hox genes suggests a link between patterning and the segmentation clock. Cell 106: 207-17.

Zhao Y, Potter SS. 2001. Functional specificity of the Hoxa13 homeobox. Development 128: 3197-207.

Zhao Y Potter SS. 2002. Functional comparison of the Hoxa 4, Hoxa 10, and Hoxa 11 homeoboxes. Dev Biol 244: 21-36. 